\title{
Geometric Complexity Identifies Platelet Activation in Familial Hypercholesterolemic Patients
}

\author{
GIORGIO BIANCIARDI, ${ }^{1 *}$ MARGHERITA AGLIANÒ,${ }^{2}$ NILA VOLPI,${ }^{2}$ AND CLAUDIA STEFANUTTI ${ }^{3}$ \\ ${ }^{1}$ Department of Medical Biotechnologies, Anatomia Patologica, University of Siena, Siena, Italy \\ ${ }^{2}$ Department of Medical Sciences, Surgery and Neurosciences, University of Siena, Siena, Italy \\ ${ }^{3}$ Department of Molecular Medicine, University of Rome "Sapienza", Roma, Italy
}

\begin{abstract}
KEYWORDS platelets; fractal analysis; platelet activation; familial hypercholesterolemia
\end{abstract}
\begin{abstract}
Familial hypercholesterolemia (FH), a genetic disease, is associated with a severe incidence of athero-thrombotic events, related, also, to platelet hyperreactivity. A plethora of methods have been proposed to identify those activated circulating platelets, none of these has proved really effective. We need efficient methods to identify the circulating platelet status in order to follow the patients after therapeutic procedures. We propose the use of computerized fractal analysis for an objective characterization of the complexity of circulating platelet shapes observed by means of transmission electron microscopy in order to characterize the in vivo hyperactivated platelets of familial hypercholesterolemic patients, distinguishing them from the in vivo resting platelets of healthy individuals. Platelet boundaries were extracted by means of automatically image analysis. Geometric complexity (fractal dimension, $D$ ) by box counting was automatically calculated. The platelet boundary observed by electron microscopy is fractal, the shape of the circulating platelets is more complex in $\mathrm{FH}(n=6)$ than healthy subjects $(n=5, P<0.01)$, with $100 \%$ correct classification in selected individuals. In vitro activated platelets from healthy subjects show an analogous increase of $D$. The observed high $D$ in the platelet boundary in $\mathrm{FH}$ originates from the in vivo platelet activation. Computerized fractal analysis of platelet shape observed by transmission electron microscopy can provide accurate, quantitative data to study platelet activation in familial hypercholesterolemia and after administration of drugs or other therapeutic procedures. Microsc. Res. Tech. 78:519-522, 2015. (C) 2015 Wiley Periodicals, Inc.
\end{abstract}

\section{INTRODUCTION}

Athero-thrombotic events are the leading cause of morbidity and mortality in the world (Labarthe and Dumbar, 2012). Familial (genetic) hypercholesterolemia $(\mathrm{FH})$ is associated with a severe incidence of those ischemic cardiovascular events and markedly enhances the risk of stroke and death (Tremoli et al., 1993). Its related increased platelet sensitivity to agonists (platelet hyperreactivity) is considered one of the major contributors to the accelerated development of atherosclerosis and thrombotic complications observed in the patients (Libby, 2002). Platelet hyperactivity is consequence of multiple pathways, e.g. the stimulation of several non-receptor protein-tyrosine kinases and increased activity of calpain, a calcium-dependent cysteine protease whose activation depends on intracellular calcium concentrations, able to activate the cytoskeletal proteins, described in different atherosclerosis-linked diseases (Bianciardi et al., 1986a; Huo et al., 2003; Trovati and Anfossi, 2002 ). Platelet shape change arises: activated platelets undergo morphologic changes, shifting from smooth disks into irregular spheroids. Platelet granules collapse, fibrinogen is produced and, eventually, platelets extrude filopodia, which not only enhances adhesion but also are rich in GP IIb/IIIa receptors and other molecules, like P-selectin, that assist in platelet-platelet interactions and platelet-endothelial cell adhesion, respectively (Israels et al., 2005; Taylor and Granger, 2003).

Recent articles have shown that in vitro activated platelets are fractals, showing a self-similar shape, when observed in light microscopy or in transmission electron microscopy (Bianciardi, 2015; Kraus et al., 2014). In these works, fractal dimension appears as an accurate descriptor of the platelet shape-change upon in vitro platelet activation by various agonists.

Here, we tested the hypothesis that computerized fractal analysis of the platelet boundaries observed at high resolution by means of transmission electron microscopy may be able to characterize the in vivo hyperactivated platelets of familial hypercholesterolemic patients, distinguishing them from the resting platelets of healthy individuals.

\footnotetext{
*Correspondence to: G. Bianciardi, Department of Medical Biotechnologies, Anatomia Patologica, Università Di Siena, via Delle Scotte 6, Siena, 53100, Italy. E-mail: giorgio.bianciardi@unisi.it

Received 9 March 2015; accepted in revised form 17 March 2015

REVIEW EDITOR: Prof. George Perry

DOI 10.1002/jemt.22503

Published online 14 April 2015 in Wiley Online Library (wileyonlinelibrary.com).
} 


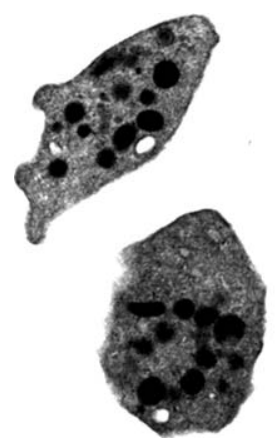

Fig. 1. Transmission electron microscopy of platelets (left) and theirs contours after thresholding (right) in a control subject $(3200 \times$, original magnification).

\section{METHODS \\ Patients}

In this study, we included six young homozygous hypercholesterolemic patients with a clinical diagnosis of $\mathrm{FH}$ and five sex- and age-matched healthy subjects. Patients with $\mathrm{FH}$ were classified by raised plasma and LDL cholesterol, the presence of tendon xanthomas, genetic analysis, and family history.

\section{Platelets}

Platelets were collected as platelet rich plasma (PRP). To obtain PRP, blood was withdrawn into a plastic syringe containing $3.8 \%$ sodium citrate (1:8) and centrifuged for $15 \mathrm{~min}$ at $100 \mathrm{~g}$ at room temperature.

\section{In Vitro Activation Study}

To perform an in vitro activation study, platelet PRP from healthy subjects $(n=5)$ was in vitro stimulated by $0.02 \mathrm{U} / \mathrm{mL}$ human thrombin (Calbiochem, Merck).

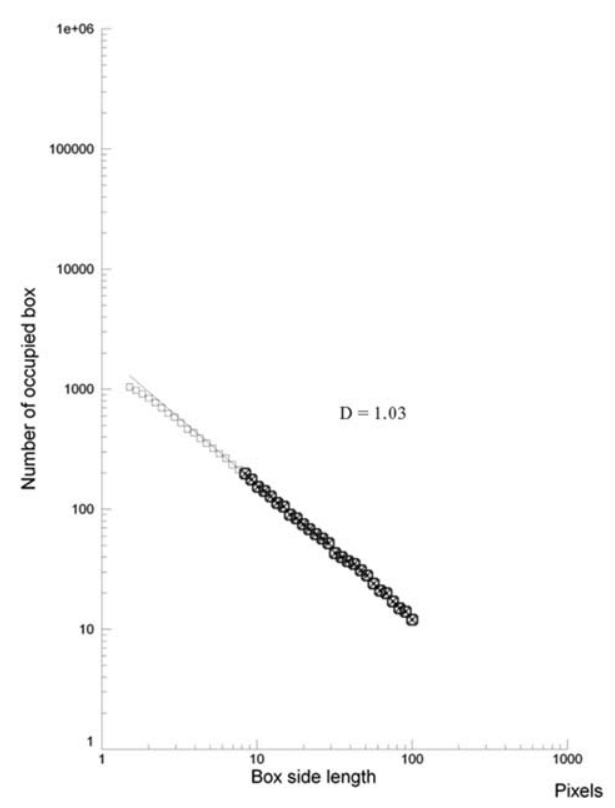

Fig. 2. Log-log plots: from a platelet of a healthy subject (left), from a platelet of a FH patient (right). The slope is the fractal dimension (geometric complexity), $D$. The linearity of the log-log plots indicates
PRP was incubated with thrombin (activated platelets) or with buffer (resting platelets) for $10 \mathrm{~min}$ at $37^{\circ} \mathrm{C}$.

\section{Electron Microscopy}

Glutaraldehyde-fixed (1.5\%) platelets were postfixed in osmium tetroxide (1\%), dehydrated by acetone, embedded in araldite, and stained with lead citrate and uranyl acetate. About hundred platelets for sample were grabbed at $\times 3,200$ without any selection.

\section{Image Analysis}

By gray level threshold segmentation, single pixel outlines of the contours of the platelets were automatically obtained (JMicroVision 1.27 software: www. microvision.com, Fig. 1).

\section{Fractal Analysis}

The local fractal dimension $(D)$ of the skeletonized image fitted in a $500 \times 500$ window was automatically measured using the box-counting algorithm (our software written in Visual Basic language). Briefly, each image was covered by a net of $L$ square boxes (from 100 to 10 pixels) and the number of boxes containing any part of the outline $N_{\mathrm{b}}(L)$ was counted. The slope of the log-log plot of $N_{\mathrm{b}}(L)$ vs. $1 / L$ represented the fractal dimension of the distribution (Falconer, 1990) (Fig. 2). The log-log straight line $(P<0.001)$ revealed the statistical self-similarity of the platelet boundaries, justifying the fractal approach.

The procedure was calibrated against shapes of known fractal dimension (square, circle and quadrich Koch island) with accuracy $\pm 2 \%$.

The analysis was reproducible, with mean intraand inter-observer coefficients of variation (CV\%) of $<2 \%$ and $<3 \%$, respectively, less than inside single

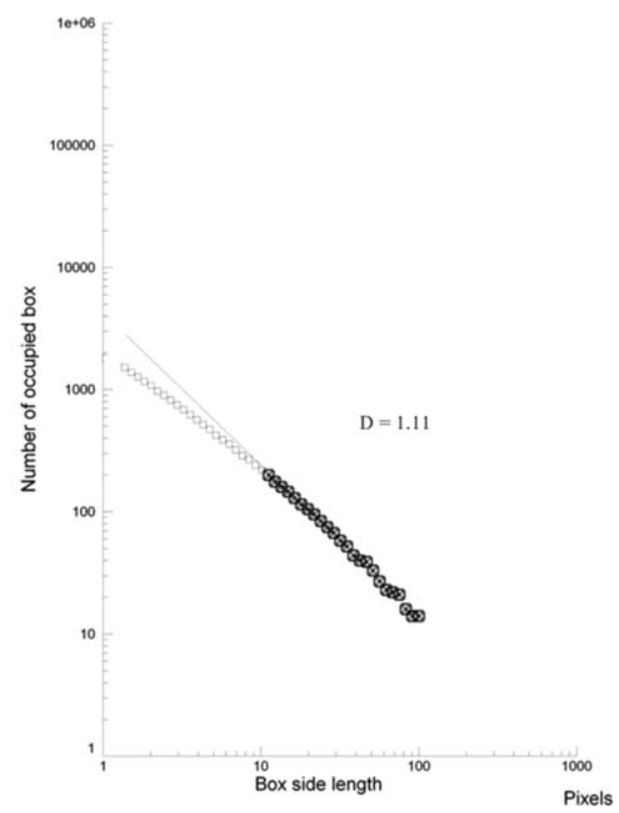

that in the scaling window used platelets are self-similar, or "fractals," in healthy subjects and in FH patients, when observed by means of transmission electron microscopy. 


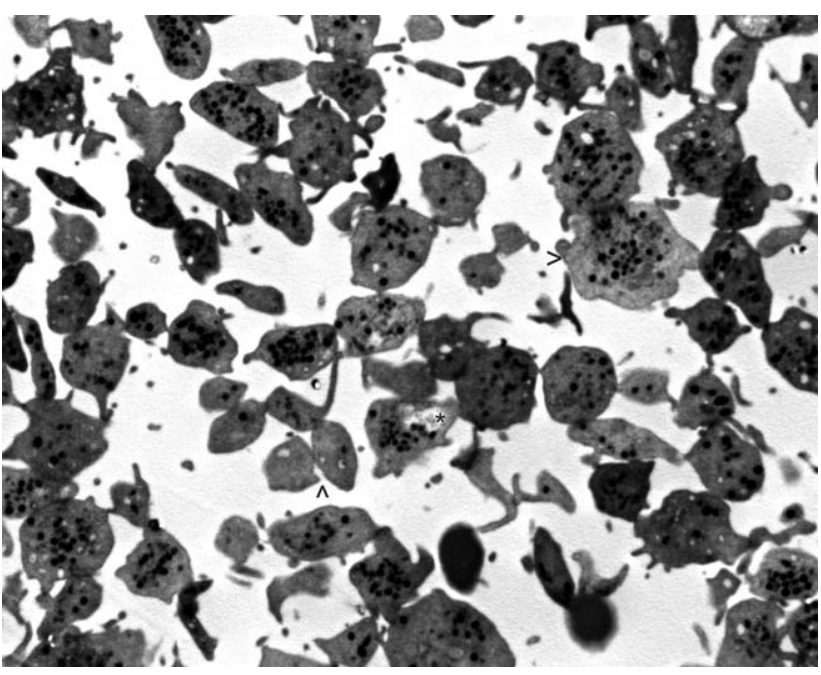

Fig. 3. Circulating platelets of a FH patient. Platelet granules collapse $(>)$, platelet degranulation $(\wedge)$, fibrinogen production $(*)$, presence of long pseudopods. Platelets adhere to each other, some microaggregates are developing. Transmission electron microscopy, $3,200 \times$.

TABLE 1. Geometric complexity (D) of platelets in healthy subjects and in familial hypercholesterolemic patients

\begin{tabular}{lll}
\hline & Mean (SD) & \\
\hline Healthy subjects $(n=5)$ & $1.03(0.011)$ & $P<0.01$ \\
FH patients $(n=6)$ & $1.10(0.012)$ & $P$
\end{tabular}

In $\mathrm{FH}$ patients $D$ values of the circulating platelets are higher than the ones of healthy subjects.

sample coefficient of variation that produced mean values of $4 \%$.

\section{Statistical Analysis}

The Kruskal-Wallis test was applied in order to verify significant differences between the groups. A linear regression analysis was applied in order to verify the significance of the log-log plot. In order to evaluate the predictive significance of fractal dimension with respect to the subjects a chi-square test was applied to the grouped cases classified by local fractal dimension $(D$ cut-off $=1.07$ ) according to the healthy or FH status.

\section{RESULTS}

Transmission electron microscopic examination of platelet samples of $\mathrm{FH}$ subjects revealed morphologies characteristic of a state of platelet hyperactivation: degranulation of platelets with extended pseudopodia, presence of platelet-rich fibrinogen, and of small microaggregates (Fig. 3).

Running the automatic fractal analysis software, circulating platelets of $\mathrm{FH}$ patients show values of $D$ higher than the one of healthy subjects $(P<0.01$, Table 1$)$

High values of $D$ were also observed in platelets of healthy individuals after in vitro activation by human thrombin $(P<0.001$, Table 2$)$.

The percentage of grouped cases classified by local fractal dimension ( $D$ cut-off $=1.07$ ) according to the subjects (FH vs. healthy) showed a $100 \%$ ratio in
TABLE 2. Geometric complexity (D) of platelets in healthy subjects, incubated with buffer ("resting platelets") or stimulated by $0.02 \mathrm{UI}$ mL human thrombin ("activated platelets")

\begin{tabular}{lll}
\hline & Mean (SD) & \\
\hline Resting platelets $(n=5)$ & $1.05(0.02)$ & \\
In vitro activated platelets $(n=5)$ & $1.16(0.02)$ & $P<0.001$ \\
\hline
\end{tabular}

In vitro stimulated platelets present $D$ values higher than the ones of resting platelets.

TABLE 3. Predicted group membership by geometric complexity (D) of blood platelets, familial hypercholesterolemic $(F H)$ patients vs. healthy subjects

\begin{tabular}{lccc}
\hline & \multicolumn{2}{c}{$\begin{array}{c}\text { Predicted group } \\
\text { membership }\end{array}$} \\
\hline Actual group & No. of cases & Healthy & $\mathrm{FH}$ \\
\hline Low $D$ & 5 & 5 & 0 \\
High $D$ & 6 & $100 \%$ & $0 \%$ \\
& & 0 & 6 \\
\end{tabular}

Percent of grouped cases correctly classified by local fractal dimension (D cut-off 51.07), according to the subjects: $100 \%$ ratio between the number of correctly classified cases and all selected cases, $P<0.001$.

selected individuals between the number of correctly classified cases and all cases, $P<0.001$ (Table 3 ).

\section{DISCUSSION}

Platelets are involved in multiple steps leading to athero-thrombosis, both in the promotion of atherosclerotic plaque growth and in the formation of thrombus on eroded or ruptured plaques (Coller, 2011). The platelet hyperreactivity, the production and platelet volume increase, both present in $\mathrm{FH}$ patients, produces a larger risk for arterial thrombosis than normocholesterolemic individuals (Davi et al., 1992)

At biochemical level, circulating platelets were demonstrated in vivo hyperactivated in $\mathrm{FH}$ condition by Carvalho et al, showing increased sensitivity to aggregating agents and increased nucleotide release levels (Carvalho et al., 1974); likewise, at morphological level, changes of the platelet plasma-membrane ultrastructure linked to in vivo platelet activation were described by us in experimental hypercholesterolemia as well in familial hypercholesterolemia, by the use of freeze-fracture ultrastructural techniques (Bianciardi et al., 1986b; Weber et al., 1978).

Since then, a plethora of abnormalities in platelet function have been described in $\mathrm{FH}$ patients, as well in other atherosclerosis-linked condition: increase of adhesion and aggregation in response to agonists, increased thromboxane production, platelet specific protein release, calcium mobilization, and adhesion molecule expression, a decrease of sensitivity to anti-aggregating agents, and changes in platelet volume, shape, life span, turnover, and membrane fluidity (Martin et al., 2012). Unfortunately, the various function tests proposed until now (light transmission aggregometry., the gold standard, multiplate whole blood aggregometry, flow cytometry, test for markers of activation on the platelet surfaces following exposure to ADP or other aggregating tests, the VerifyNow assay that detects whole blood platelet aggregation using optical detection) are not able to assess the real status of platelets in the blood stream (Michelson, 2009). 
In pathology, fractal dimension, the critical exponent characterizing the geometric complexity of fractal objects, has been shown to be capable of performing diagnosis and prognosis in human diseases (Bianciardi et al., 2003, 2013; Cross and Cotton, 1992; Cross et al., 1994; Goldberger and West, 1987; Losa and Nonnenmacher, 1996). In effect, anatomical entities show complexity as a basic characteristic, residing in the structure and in the behavior of the cell, organ, and apparatus (Grizzi and Chriva-Internati, 2005). Matching the variety of complex natural objects, Mandelbrot created a new language to describe them, the so-called "fractal geometry" (Mandelbrot, 1983). In particular, where surface phenomena are of crucial importance, a number of complex anatomic structures display fractallike properties (Goldberger and West, 1987). The recent articles by Kraus et al. and Bianciardi (Bianciardi, 2015; Kraus et al., 2014), characterizing the platelets by light microscopy or transmission electron microscopy, and the present article performed by transmission electron microscopy shows that platelets display selfsimilarity, or, in other words, they are fractals.

In the present article, also, fractal analysis has been tested in order to search for a different geometric complexity of the circulating platelets in patients with $\mathrm{FH}$ in comparison to normal healthy subjects by using transmission electron microscopy in order to have a high resolution of the cells. We first demonstrated that in patients with $\mathrm{FH}$, platelets present a higher value of geometric complexity than in healthy subjects that shall be linked to the in vivo hyperactivation status of the FH patients, being possible to reproduce an analogous feature by the in vitro activation of platelets collected from healthy subjects.

Our fractal approach was able to distinguish accurately between subjects with a $100 \%$ correct classification in selected individuals, giving us a new approach to objectively and accurately quantify the status of platelets in familial, genetically determined, hypercholesterolemia. This method, that may be also performed by using commercial software like the Benoit 1.3 software, TruSoft Int'l Inc: http://trusoft-international. com/benoit.html), may be promising to study circulating platelets in other pathophysiological condition linked to platelet activation and after administration of drugs or other therapeutic procedures (Stefanutti et al., 2012; Stefanutti and Julius, 2013).

\section{ACKNOWLEDGMENT}

This research article was developed within the frame of the Multidisciplinary International Group for Hemapheresis TherapY and MEtabolic DIsturbances Contrast (MIGHTY MEDIC). http://www.mightymedic.org

\section{REFERENCES}

Bianciardi G. 2015. Geometric complexity is increased in in vitro activated platelets. Microsc Res Tech, Mar 22. doi: 10.1002/jemt.22498. [Epub ahead of print]
Bianciardi G, Vittoria A, Toti P, Franchi M, Martelli G, Di Perri T, Weber G. 1986a. Ultrastructural modifications of the platelet plasma-membrane in vascular diseases and the effect of carbochromen derivative as studied by freeze-fracture and computer measurement. Atherosclerosis 62:173-178.

Bianciardi G, Weber G, Toti P, Widhalm K, Sinzinger H. 1986b. Platelet plasma-membrane changes in human type IIa hypercholesterolemia. Appl Pathol 4:253-259.

Bianciardi G, Miracco C, De Santi MM, Luzi P. 2003. Differential diagnosis between mycosis fungoides and chronic dermatitis by fractal analysis. J Dermatol Sci 33:184-86.

Bianciardi G, Miracco C, Lazzi S, Luzi P. 2013. Fractal analysis of epithelial-connective tissue interface in basal cell carcinoma of the skin. Curr Bioinformatics 8:357-361.

Carvalho ACA, Colman RW, Lees RS. 1974. Platelet function in hypercholesterolemia. N Engl J Med 290:434-438.

Coller BS. 2011. Historical perspective and future directions on platelet research. J Thromb Haemost 9 (Suppl 1):374-395.

Cross SS, Cotton DWK. 1992. The fractal dimension may be a useful morphometric discriminant in histopathology. J Pathol 166:409411.

Cross SS, Bury JP, Silcocks PB, Stephenson TJ, Cotton DW. 1994. Fractal geometric analysis of colorectal polyps. J Pathol 172:317323.

Davi G, Averna M, Catalano I, Barbagallo C, Ganci A, Notarbartolo A, Ciabattoni G, Patrono C. 1992. Increased thromboxane biosynthesis in type IIa hypercholesterolemia. Circulation 85:17921798.

Falconer K. 1990. Fractal geometry: Mathematical foundations and applications. Chichester, New York: Wiley.

Goldberger AL, West AJ. 1987. Fractals in physiology and medicine. Yale J Biol Med 60:421-435.

Grizzi F, Chriva-Internati M. 2005. The complexity of anatomical system. Theor Biol Med Model 2:26-34.

Huo Y, Schober A, Forlow SB, Smith DF, Hyman MC, Jung S. 2003. Circulating activated platelets exacerbate atherosclerosis in mice deficient in apolipoprotein E. Nat Med 9:61-67.

Israels SJ, et al. 2005. cd63 modulates spreading and tyrosine phosphorylation of platelets on immobilized fibrinogen. Thromb Haemost 93:311-318.

Kraus M-J, et al. 2014. Fractal and Euclidean descriptors of platelet shape. Platelets 25:488-498.

Labarthe DR, Dunbar SB. 2012. Global cardiovascular health promotion and disease prevention: 2011 and beyond. Circulation 125: 2667-2676.

Libby P. 2002. Inflammation in atherosclerosis. Nature 420:868-874.

Losa GA, Nonnenmacher TF. 1996. Self-similarity and fractal irregularity in pathologic tissues. Mod Pathol 9:174-182.

Mandelbrot BB. 1983. The fractal geometry of nature. New York: Freeman.

Martin JF, Kristensen SD, Mathur A, Grove EL, Choudry FA. 2012. The causal role of megakaryocyte-platelet hyperactivity in acute coronary syndromes. Nat Rev Cardiol 9:658-670.

Michelson AD. 2009. Methods for the measuremt of platelet function. Am J Cardiol 103:20A-26A.

Stefanutti C, Julius U. 2013. Lipoprotein apheresis: State of the art and novelties. Atheroscler Suppl 14:19-27.

Stefanutti C, et al. 2012. New clinical perspectives of hypolipidemic drug therapy in severe hypercholesterolemia. Curr Med Chem 19: 4861-4868.

Taylor A, Granger DN. 2003. Hypercholesterolemia promotes Pselectin-dependent platelet-endothelial cell adhesion in postcapillary venules. Arterioscler Thromb Vasc Biol 23:675-680.

Tremoli E, Colli S, Maderna P, Baldassarre D, Di Minno G. 1993. Hypercholesterolemia and platelets. Sem Thromb Hemost 19:115121.

Trovati M, Anfossi G. 2002. Mechanisms involved in platelet hyperactivation and Platelet-endothelium interrelationships in diabetes mellitus. Curr Diabetes Rep 2:316-322.

Weber G, Bianciardi G, Pierli C. 1978. Preliminary observations on the ultrastructural modifications of the freeze-cleaved circulating platelets surface in hypercholesterolemic rabbits treated with AD6. Pharmacol Res Comm 10:739-746. 